\title{
A Pre-aggregation Fuzzy Collaborative Intelligence- based Fuzzy Analytic Hierarchy Process Approach for Selecting Alternative Suppliers amid the COVID-19 Pandemic
}

\author{
Tin-Chih Toly Chen ${ }^{1, *}$, Hsin-Chieh Wu ${ }^{2}$ \\ 1 Department of Industrial Engineering and Management, National Chiao Tung University, 1001, University \\ Road, Hsinchu 30010, Taiwan \\ 2 Department of Industrial Engineering and Management, Chaoyang University of Technology, Taichung \\ 413310, Taiwan; hcwul@cyut.edu.tw \\ * Correspondence: tolychen@ms37.hinet.net
}

\begin{abstract}
In the existing group decision-making fuzzy analytic hierarchy process (FAHP) methods, the consensus among experts has rarely been fully reached. To fill this gap, in this study, a preaggregation fuzzy collaborative intelligence (FCI)-based FAHP approach is proposed. In the proposed pre-aggregation FCI-based FAHP approach, fuzzy intersection is applied to aggregate experts' pairwise comparison results if these pairwise comparison results overlap. The aggregation result is a matrix of polygonal fuzzy numbers. Subsequently, alpha-cut operations are applied to derive the fuzzy priorities of criteria from the aggregation result. The pre-aggregation FCI-based FAHP approach has been applied to select suitable alternative suppliers for a wafer foundry in Taiwan amid the COVID-19 pandemic. The experimental results revealed that the pre-aggregation FCI-based FAHP approach significantly reduced the uncertainty inherent in the decision-making process by deriving fuzzy priorities with very narrow ranges.
\end{abstract}

Keywords: group decision-making; fuzzy analytic hierarchy process; consensus; wafer foundry; COVID-19 pandemic

\section{Introduction}

Analytic hierarchy process (AHP), proposed by Saaty [1], is a popular multiple-criteria decisionmaking method. AHP is based on pairwise comparisons made by an expert. However, pairwise comparisons are subjective and the expert is sometimes not sure of the results. To address this issue, various fuzzy AHP (FAHP) methods, such as fuzzy geometric mean (FGM) [2], fuzzy extent analysis (FEA) [3], and alpha-cut operations (ACO) [4], have been proposed by expressing pairwise comparison results with fuzzy sets. The types of fuzzy sets adopted in these methods include ordinary fuzzy sets [5], intuitionistic fuzzy sets [6], interval type-2 fuzzy sets [7], hesitant fuzzy sets [8], etc.

Multiple experts are usually involved in a FAHP problem to avoid personal bias and omissions, which turns the FAHP problem into a group decision-making FAHP problem [9]. Sometimes the pairwise comparison results by experts are aggregated before they derived the fuzzy priorities of criteria, i.e., the pre-aggregation way [5,10-12]. There are also studies in which the fuzzy priorities of criteria derived by experts are aggregated instead, i.e., the post-aggregation way [13-16], is to aggregate the fuzzy priorities of criteria derived by experts. The pre-aggregation way eliminates the 
necessity for each expert to derive the fuzzy priorities of criteria individually, while the postaggregation way is easier to calculate. This study follows the pre-aggregation way.

The existing pre-aggregation FAHP methods are subject to the following problems:

(1) Experts' judgment are usually aggregated without checking whether they have reached a consensus [5].

(2) Most past studies aggregated experts' judgments using FGM [5,11], which may lead to unreasonable and unacceptable results [17].

(3) A few studies measured the distance between any two experts' judgments to check the existence of a consensus [10]. However, distance-based measures increase the uncertainty of the fuzzy group decision-making process, because a suitable distance function needs to be chosen, and a threshold for two judgments' distance has to be established.

Fuzzy collaborative intelligence (FCI) methods [18-19] adopt fuzzy intersection (FI) as the aggregator and can solve these problems naturally [20]. However, FCI methods have only been applied to post-aggregation problems [15-16] because the calculation is easier. To fill this gap, in this study, a pre-aggregation FCI-based FAHP approach is proposed.

In the proposed pre-aggregation FCI-based FAHP approach, FI is applied to aggregate experts' pairwise comparison results. If all FI outcomes are non-empty sets, then there is a consensus among experts; otherwise, experts need to modify their pairwise comparison results. Subsequently, considering the special shapes of FI outcomes, alpha-cut operations (ACO) [21] are applied to derive the fuzzy priorities of criteria from the aggregation result.

The difference between the proposed methodology and some existing pre-aggregation group decision-making FAHP methods are listed in Table 1. The proposed methodology has been applied to an alternative supplier selection problem amid the COVID-19 pandemic.

Table 1. Differences between the proposed methodology and some existing group decision-making FAHP methods.

\begin{tabular}{cccccc}
\hline Method & $\begin{array}{c}\text { Consensus } \\
\text { Measure }\end{array}$ & $\begin{array}{c}\text { Level of } \\
\text { Consensus }\end{array}$ & $\begin{array}{c}\text { Aggregation } \\
\text { Method }\end{array}$ & $\begin{array}{c}\text { Method for } \\
\text { Deriving Fuzzy } \\
\text { Priorities }\end{array}$ & $\begin{array}{c}\text { Acceptability } \\
\text { of Results }\end{array}$ \\
\hline $\begin{array}{c}\text { Zheng et al. } \\
{[5]}\end{array}$ & No & Unknown & Discussion & FGM & Unknown \\
\hline $\begin{array}{c}\text { Wang et al. } \\
{[11]}\end{array}$ & No & Low & FGM & FEA & Low High \\
\hline $\begin{array}{c}\text { Gao et al. [10] } \\
\text { Distance between } \\
\text { judgments }\end{array}$ & Moderate & FGM & FGM & $\begin{array}{c}\text { Moderate } ~ \\
\text { High }\end{array}$ \\
\hline $\begin{array}{c}\text { The proposed } \\
\text { methodology }\end{array}$ & FI result & High & FI & ACO & High \\
\hline
\end{tabular}

The remainder of this paper is organized as follows. Section 2 reviews the existing methods for measuring the consensus among experts in an FAHP application. Section 3 introduces the preaggregation FCI-based FAHP approach. Section 4 reports the results of applying the pre-aggregation FCI-based FAHP approach to select suitable alternative suppliers for a wafer foundry in Taiwan amid the COVID-19 pandemic. Some existing methods were also applied to this case for comparison. Section 5 concludes this study and puts forth some potential topics for future research.

\section{Existing Consensus Measures in Group Decision-making FAHP}

Most past FAHP studies did not check the existence of a consensus among experts, but directly aggregated the pairwise comparison results done by experts or the fuzzy priorities of criteria derived by them [15], which may lead to unacceptable results [17]. There are two time points at which the consensus among experts can be measured: before deriving the fuzzy priorities of criteria (i.e., the 
pre-aggregation way), and after deriving the fuzzy priorities of criteria (i.e., the post-aggregation way) [22].

In the pre-aggregation way, a consensus exists if the distance between the fuzzy judgment matrixes of any two experts is smaller than a threshold $\tilde{\xi}$ [10],

$$
\tilde{d}(\tilde{\mathbf{A}}(k), \tilde{\mathbf{A}}(l))=\sqrt{\sum_{i=1}^{n} \sum_{j=1}^{n}\left(\tilde{a}_{i j}(k)(-) \tilde{a}_{i j}(l)\right)^{2}} \leq \tilde{\xi} \quad \forall k \neq l
$$

where $\tilde{d}()$ is the fuzzy distance function; $\tilde{\mathbf{A}}(k)=\left[\tilde{a}_{i j}(k)\right]$ is the fuzzy judgment matrix of expert $k$; $\tilde{a}_{i j}(k)$ indicates the relative priority of criterion $i$ over criterion $j$ compared by expert $k ; k=1 \sim K$. A similar condition is to request the distance from the fuzzy judgment matrix of each expert to the average of all experts' fuzzy judgment matrixes to be smaller than the threshold:

$$
\tilde{d}(\tilde{\mathbf{A}}(k), \tilde{\mathbf{A}}(\text { all })) \leq \tilde{\xi} \quad \forall k
$$

where $\tilde{\mathbf{A}}($ all $)=\left[\tilde{a}_{i j}(\right.$ all $\left.)\right]$ is the average (or geometric mean) of all experts' fuzzy judgment matrixes:

$$
\tilde{a}_{i j}(a l l)=\frac{\sum_{k=1}^{K} \tilde{a}_{i j}(k)}{K} \text { or } \sqrt[K]{\prod_{k=1}^{K} \tilde{a}_{i j}(k)}
$$

In the post-aggregation way, a consensus exists if the distance between the fuzzy priorities derived by any two experts is smaller than a threshold $\tilde{\zeta}$,

$$
\tilde{d}(\tilde{\mathbf{w}}(k), \tilde{\mathbf{w}}(l))=\sqrt{\sum_{i=1}^{n}\left(\tilde{w}_{i}(k)(-) \tilde{w}_{i}(l)\right)^{2}} \leq \tilde{\zeta} \quad \forall k \neq l
$$

where $\tilde{\mathbf{w}}(k)$ is the fuzzy priorities of criteria derived by expert $k$. A similar condition is to request or the distance from the fuzzy priorities derived by each expert to the average of fuzzy priorities derived by all experts to be smaller than the threshold:

$$
\tilde{d}(\tilde{\mathbf{w}}(k), \tilde{\mathbf{w}}(\text { all })) \leq \tilde{\zeta} \quad \forall k
$$

where $\tilde{\mathbf{w}}($ all $)=\left[\tilde{w}_{i}(\right.$ all $\left.)\right]$ is the average of fuzzy priorities derived by all experts:

$$
\tilde{w}_{i}(a l l)=\frac{\sum_{k=1}^{K} \tilde{w}_{i}(k)}{K}
$$

Distance-based criteria are subject to the following problems:

(1) The formula for calculating the distance between two fuzzy judgment matrixes (or fuzzy priorities) is not easy to calculate.

(2) There are other methods to measure the distance between two fuzzy judgment matrixes (or fuzzy priorities). However, there is no absolute rule for choosing the most suitable method.

(3) The value of the threshold $\tilde{\xi}$ (or $\tilde{\zeta}$ ) is subjectively determined, which adds to the uncertainty of the decision-making process.

From the viewpoint of fuzzy collaborative intelligence, if experts' fuzzy judgment matrixes overlap, i.e., the FI of these fuzzy judgment matrixes is not an empty set, then there is a consensus among them [20]:

$$
F I\left(\left\{\tilde{a}_{i j}(k)\right\}\right) \neq \varnothing \quad \forall i, j, k
$$

As an alternative, if the fuzzy priorities derived by experts overlap, then there is a consensus among them:

$$
F I\left(\left\{\tilde{w}_{i}(k)\right\}\right) \neq \varnothing \quad \forall i, k
$$

Most past studies [23-24] checked the existence of a consensus among experts in the post-aggregation way using Criterion (8) to simplify the calculation. To fill this gap, in this study, the existence of a consensus among experts is checked in the pre-aggregation way using Criterion (7). 


\section{Methodology}

\subsection{Anterior Aggregation}

The fuzzy judgement matrix of expert $k$ is denoted by $\tilde{\mathbf{A}}(k)=\left[\tilde{a}_{i j}(k)\right]$. FI [20] is applied to aggregate all experts' fuzzy judgment matrixes into a consolidated fuzzy judgment matrix $\tilde{\mathbf{A}}(a l l)=\left[\tilde{a}_{i j}(a l l)\right]:$

$$
\tilde{\mathbf{A}}(\text { all })=F I(\{\tilde{\mathbf{A}}(k)\}
$$

or

$$
\tilde{a}_{i j}(a l l)=F I\left(\left\{\tilde{a}_{i j}(k)\right\}\right.
$$

with the following membership function:

$$
\mu_{\tilde{a}_{i j}(a l l)}(x)=\min _{k}\left(\mu_{\tilde{a}_{j}(k)}(x)\right)
$$

If $\tilde{a}_{i j}($ all $)$ is an empty set for any $i$ and $j$, then experts lack a consensus and need to modify their pairwise comparison results.

Without loss of generality, all fuzzy parameters and variables in the proposed methodology are given in or approximated with triangular fuzzy numbers (TFNs). For example,

$$
\tilde{a}_{i j}(k)=\left(a_{i j 1}(k), a_{i j 2}(k), a_{i j 3}(k)\right)
$$

The following theorem holds.

Theorem 1. A consensus among all experts exists if $\max _{i \neq j}\left(\max _{k \neq l}\left(\max \left(a_{i j 1}(k), a_{i j 1}(l)\right)-\min \left(a_{i j 3}(k), a_{i j 3}(l)\right)\right)\right) \leq 0$

Proof of Theorem 1. Experts reach an overall consensus regarding the value of $\tilde{a}_{i j}$ if

$$
F I\left(\left\{\tilde{a}_{i j}(l)\right\}\right) \neq \varnothing
$$

which means

$$
\left[a_{i j 1}(k), a_{i j 3}(k)\right] \cap\left[a_{i j 1}(l), a_{i j 3}(l)\right] \neq \varnothing \quad \forall k \neq l
$$

which is equivalent to

$$
\max \left(a_{i j 1}(k), a_{i j 1}(l)\right) \leq \min \left(a_{i j 3}(k), a_{i j 3}(l)\right) \quad \forall k \neq l
$$

which can be condensed into

$$
\max _{k \neq l}\left(\max \left(a_{i j 1}(k), a_{i j 1}(l)\right)-\min \left(a_{i j 3}(k), a_{i j 3}(l)\right)\right) \leq 0
$$

which holds for all $i$ and $j$. Therefore,

$$
\max _{i \neq j}\left(\max _{k \neq l}\left(\max \left(a_{i j 1}(k), a_{i j 1}(l)\right)-\min \left(a_{i j 3}(k), a_{i j 3}(l)\right)\right)\right) \leq 0
$$

Theorem 1 is proved.

The FI result of TFNs is a polygonal fuzzy number [20]. As a result, all elements of the fuzzy consolidated judgment matrix are polygonal fuzzy numbers, as illustrated in Figure 1. In addition, since FI works on the membership rather than the value of each element, the multiplicity property still holds:

$$
F I\left(\left\{\tilde{a}_{i j}(k)\right\}\right)=\frac{1}{F I\left(\left\{\tilde{a}_{j i}(k)\right\}\right)}
$$




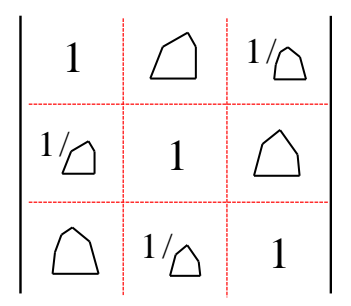

Figure 1. A consolidated fuzzy judgment matrix with polygonal fuzzy numbers.

\subsection{Deriving the Fuzzy Priorities of Criteria}

ACO [24] are applied to derive the fuzzy priorities of criteria. First, fuzzy parameters and variables are represented with their $\alpha$ cuts. For example,

$$
a_{i j}(\text { all })(\alpha)=\left[a_{i j}^{L}(\text { all })(\alpha), a_{i j}^{R}(a l l)(\alpha)\right]
$$

where $L$ and $R$ indicate the left and right $\alpha$ cuts of a fuzzy variable, respectively. Then, an eigen analysis is conducted to derive the values of fuzzy eigenvalue $\tilde{\lambda}($ all $)$ and fuzzy vector $\tilde{\mathbf{x}}($ all $)$, in terms of their $\alpha$ cuts, as

$$
\begin{gathered}
\operatorname{det}\left(\mathbf{A}^{*}(\text { all })(\alpha)-\lambda^{\dagger}(\text { all })(\alpha) \mathbf{I}\right)=0 \\
\left(\mathbf{A}^{*}(\text { all })(\alpha)-\lambda^{\dagger}(\text { all })(\alpha) \mathbf{I}\right) \mathbf{x}^{\ominus}(\text { all })(\alpha)=0
\end{gathered}
$$

where $*, \dagger$, and $\diamond$ can be $L$ or $R$. If $\alpha$ takes 11 possible values $(0,0.1, \ldots, 1)$, Equations (20) and (21) need to be solved $10 \cdot 2^{\mathrm{C}_{2}^{n}}+1$ times. The minimal and maximal values of the results are used to construct the $\alpha$ cut of fuzzy eigenvector and eigenvalue [4]:

$$
\begin{aligned}
x_{i}(\text { all })(\alpha) & =\left[x_{i}^{L}(\text { all })(\alpha), x_{i}^{R}(\text { all })(\alpha)\right] \\
& =\left[\min _{\diamond} x_{i}^{(}(\text {all })(\alpha), \max _{\diamond} x_{i}^{\diamond}(\text { all })(\alpha)\right] \\
\lambda(\text { all })(\alpha) & =\left[\lambda^{L}(\text { all })(\alpha), \lambda^{R}(\text { all })(\alpha)\right] \\
& =\left[\min _{\dagger} \lambda^{\dagger}(\text { all })(\alpha), \max _{\dagger} \lambda^{\dagger}(\text { all })(\alpha)\right]
\end{aligned}
$$

In addition, Equations (20) and (21) involve a number of fuzzy multiplication operations. As a result, if $\tilde{a}_{i j}($ all $)$ is a TFN, then $\tilde{\lambda}($ all $)$ and $\tilde{\mathbf{x}}($ all $)$ will be triangular fuzzy numbers with curved edges [21]. If $\tilde{a}_{i j}$ (all) is a polygonal fuzzy number, then $\tilde{\lambda}($ all $)$ and $\tilde{\mathbf{x}}($ all $)$ will be polygonal fuzzy numbers with curved edges.

The fuzzy priorities of criteria $\tilde{\mathbf{w}}($ all $)$ are obtained by normalizing $\tilde{\mathbf{x}}($ all $)$ as

$$
\tilde{w}_{i}(\text { all })=\frac{\tilde{x}_{i}(\text { all })}{\sum_{l=1}^{n} \tilde{x}_{l}(\text { all })}
$$

In addition, there are $n$ possible values of $\tilde{\lambda}(a l l)$. The fuzzy maximal eigenvalue is denoted by $\tilde{\lambda}_{\max }($ all $)$. The consistency of all experts' pairwise comparison results is evaluated in terms of fuzzy consistency ratio $C R($ all $)$ as

$$
C R(\text { all })=\frac{\frac{\tilde{\lambda}_{\max }(\text { all })-n}{n-1}}{R I}
$$

where $R I$ is random consistency index [1]. $C R($ all $) \geq 0 . C R($ all $)$ needs to be less than 0.1 , but can be relaxed to less than 0.3 if the problem size is large. Otherwise, experts are asked to modify their pairwise comparison results.

\section{Case Study}




\subsection{Application of the Proposed Methodology}

Supplier selection is a multiple-criteria decision-making problem that has been extensively studied [25-27]. A number of factors are considered critical to the performance of a supplier, such as product quality, price, delivery speed, company reputation, supplier relationship, etc. [15,28]. An alternative supplier is a supplier with which a formal cooperation relationship does not exist. An alternative supplier may not be the best choice in regular supplier selection, but has to be resorted to during the COVID-19 pandemic. For this reason, criteria for choosing an alternative supplier may be different from those for choosing a regular supplier [29]. In addition, amid the COVID-19 pandemic, a manufacturer (including supplier and alternative supplier) has to be robust to the pandemic to ensure its operations [16,30]. Factors critical to the robustness of a manufacturer to the COVID-19 pandemic include pandemic containment performance, pandemic severity, vaccine acquisition speed, demand shrinkage, supplier impact, and infection risk [16].

The COVID-19 pandemic has forced many factories to shut down, resulting in the breakage of related supply chains [31]. To fill the shortage of supply, a manufacturer needs to find alternative suppliers [32]. Therefore, how to choose suitable alternative suppliers amid the COVID-19 pandemic becomes an important problem. A wafer foundry in Taiwan would like to choose suitable alternative suppliers amid the COVID-19 pandemic. Current suppliers of the wafer foundry were mostly located in Taiwan, Netherlands, and USA. Among these regions, Netherlands and USA have been highly impacted by the pandemic [33]. Therefore, looking for alternative suppliers to replace existing suppliers in the two regions became a critical task to the wafer foundry. To fulfill this task, the proposed methodology was applied.

The following factors were considered critical to the performance of an alternative supplier amid the COVID-19 pandemic:

- price,

- delivery speed,

- company reputation,

- pandemic containment performance, and

- pandemic severity.

Three experts were involved in the alternative supplier selection process. At first, each expert was requested to compare the relative fuzzy priorities of criteria in pairs. The comparison results are presented below:

$$
\begin{aligned}
\tilde{\mathbf{A}}(1) & =\left[\begin{array}{ccccc}
1 & - & - & - & - \\
(5,7,9) & 1 & (3,5,7) & - & - \\
(1,3,5) & - & 1 & - & - \\
(3,5,7) & (1,3,5) & (3,5,7) & 1 & (2,4,6) \\
(1,3,5) & (1,1,3) & (1,1,3) & - & 1
\end{array}\right] \\
\tilde{\mathbf{A}}(2) & =\left[\begin{array}{ccccc}
1 & - & - & - & - \\
(2,4,6) & 1 & (3,5,7) & - & - \\
(1,1,3) & - & 1 & - & - \\
(4,6,8) & (2,4,6) & (2,4,6) & 1 & (3,5,7) \\
(2,4,6) & (1,3,5) & (1,3,5) & - & 1
\end{array}\right] \\
\tilde{\mathbf{A}}(3) & =\left[\begin{array}{ccccc}
1 & - & - & - & - \\
(3,5,7) & 1 & (2,4,6) & - & - \\
(1,2,4) & - & 1 & - & - \\
(2,4,6) & (1,2,4) & (1,3,5) & 1 & (2,4,6) \\
(1,3,5) & (1,2,4) & (1,3,5) & - & 1
\end{array}\right]
\end{aligned}
$$

Fuzzy intersection was applied to aggregate all experts' pairwise comparison results. The results are shown in Figure 2. Obviously, experts achieved a consensus regarding the value of each relative priority. 

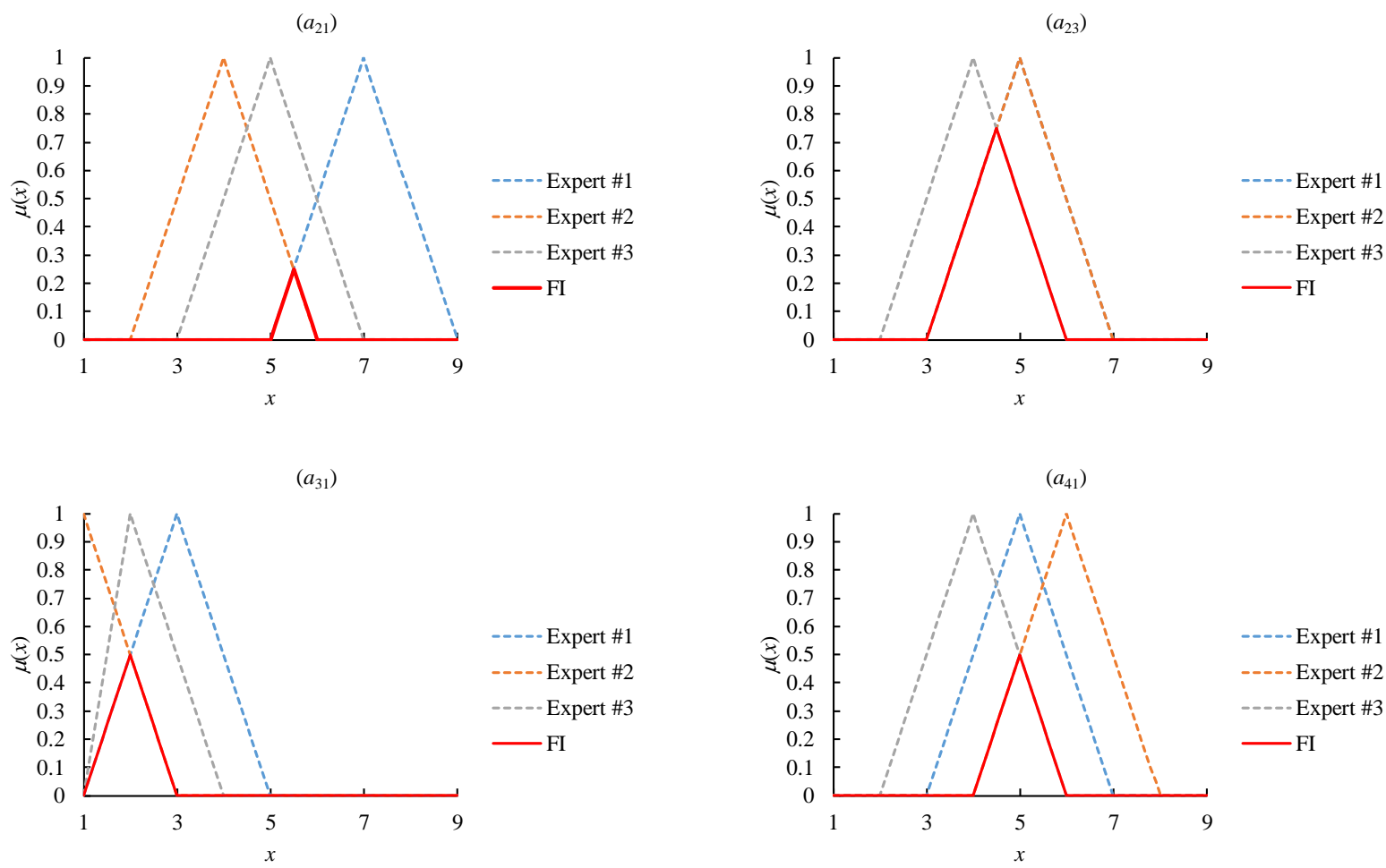

----- Expert \#1

-.--. Expert \#2

----- Expert \#3

- FI

$\left(a_{42}\right)$

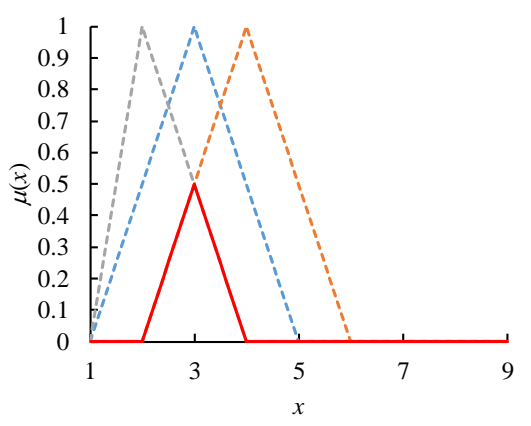

----- Expert \#1

----- Expert \#2

----- Expert \#3

- FI

$\left(a_{45}\right)$

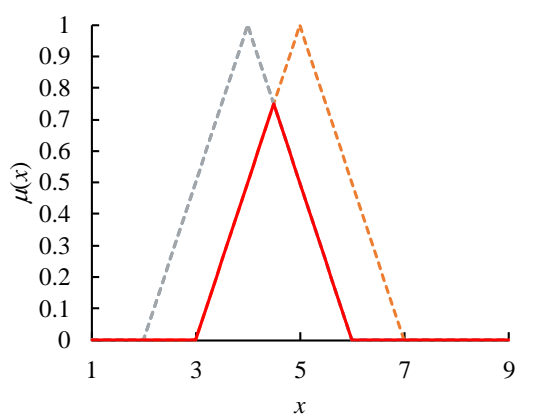

----- Expert \#1

----- Expert \#2

----- Expert \#3

- FI
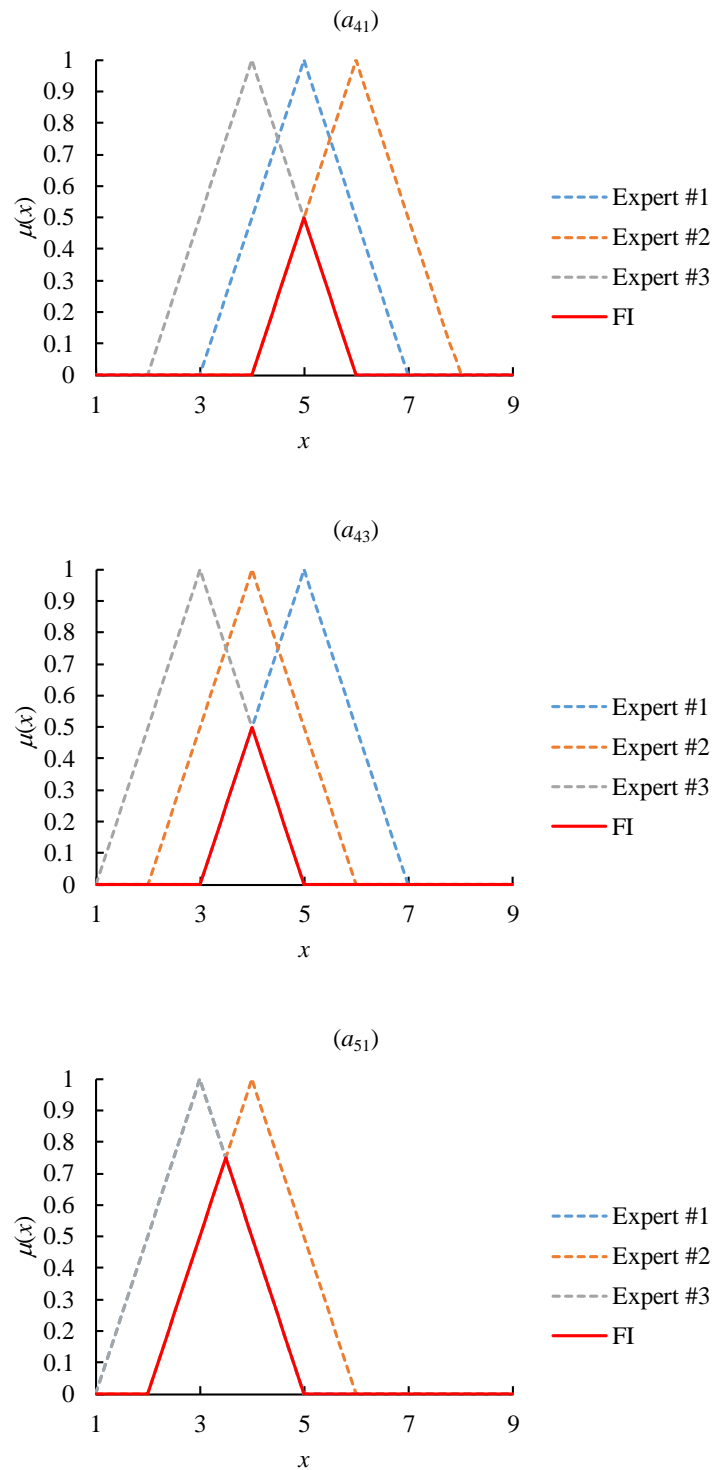

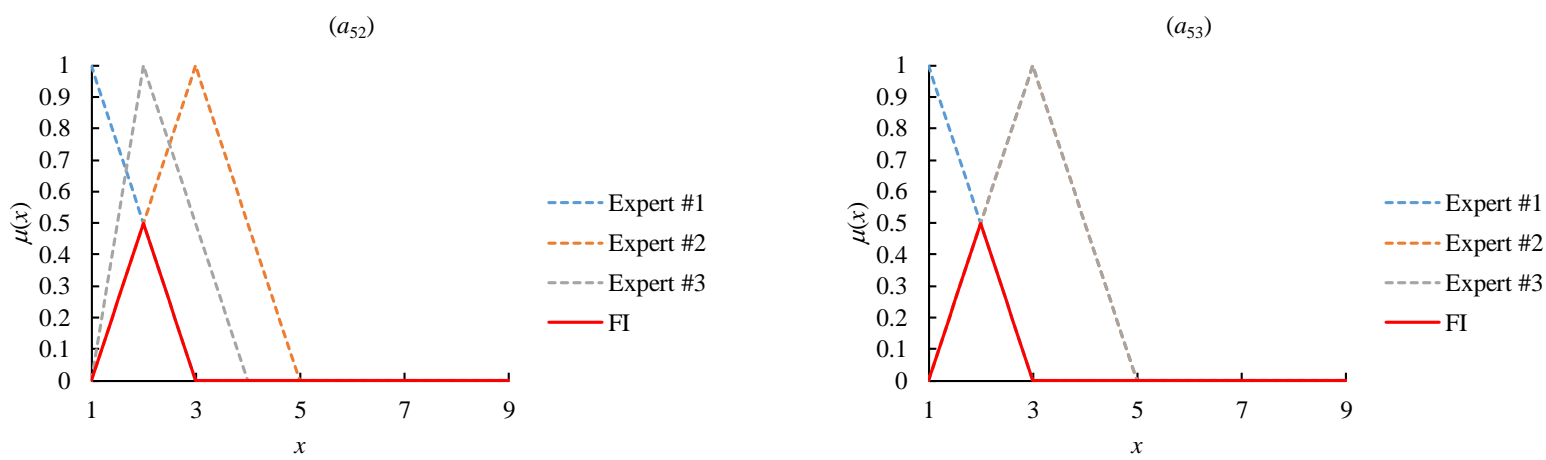

Figure 2. The fuzzy intersection results.

To apply ACO to derive the values of fuzzy priorities, the aggregation result was discretized into $\alpha$ cuts, as shown in Table 2. Obviously, only $\alpha$ cuts when $\alpha$ was less than 0.2 needed to be considered, which significantly shortened the enumeration process. Subsequently, MATLAB 2017 was adopted to implement ACO on a PC with i7-7700 CPU 3.6 GHz and 8 GB RAM. The program code was shown in Figure 3. The execution time was 6.4 seconds. Finally, the derived fuzzy maximal eigenvalue and fuzzy priorities of criteria are shown in Figures 4 and 5, respectively.

Table 2. The $\alpha$ cuts of the aggregation result.

\begin{tabular}{|c|c|c|c|c|c|c|c|c|c|c|}
\hline$\alpha$ & $a_{21}$ & $a_{23}$ & $a_{31}$ & $a_{41}$ & $a_{42}$ & $a_{43}$ & $a_{45}$ & $a_{51}$ & $a_{52}$ & $a_{53}$ \\
\hline 0 & {$[5,6]$} & {$[3,6]$} & {$[1,3]$} & {$[4,6]$} & {$[2,4]$} & {$[3,5]$} & {$[3,6]$} & {$[2,5]$} & {$[1,3]$} & {$[1,3]$} \\
\hline 0.1 & {$[5.2,5.8]$} & {$[3.2,5.8]$} & {$[1.2,2.8]$} & {$[4.2,5.8]$} & {$[2.2,3.8]$} & {$[3.2,4.8]$} & {$[3.2,5.8]$} & {$[2.2,4.8]$} & {$[1.2,2.8]$} & {$[1.2,2.8]$} \\
\hline 0.2 & {$[5.4,5.6]$} & {$[3.4,5.6]$} & {$[1.4,2.6]$} & {$[4.4,5.6]$} & {$[2.4,3.6]$} & {$[3.4,4.6]$} & {$[3.4,5.6]$} & {$[2.4,4.6]$} & {$[1.4,2.6]$} & {$[1.4,2.6]$} \\
\hline 0.3 & & {$[3.6,5.4]$} & {$[1.6,2.4]$} & {$[4.6,5.4]$} & {$[2.6,3.4]$} & {$[3.6,4.4]$} & {$[3.6,5.4]$} & {$[2.6,4.4]$} & {$[1.6,2.4]$} & {$[1.6,2.4]$} \\
\hline 0.4 & & {$[3.8,5.2]$} & {$[1.8,2.2]$} & {$[4.8,5.2]$} & {$[2.8,3.2]$} & {$[3.8,4.2]$} & {$[3.8,5.2]$} & {$[2.8,4.2]$} & {$[1.8,2.2]$} & {$[1.8,2.2]$} \\
\hline 0.5 & & {$[4,5]$} & {$[2,2]$} & {$[5,5]$} & {$[3,3]$} & {$[4,4]$} & {$[4,5]$} & {$[3,4]$} & {$[2,2]$} & {$[2,2]$} \\
\hline 0.6 & & {$[4.2,4.8]$} & & & & & {$[4.2,4.8]$} & {$[3.2,3.8]$} & & \\
\hline 0.7 & & {$[4.4,4.6]$} & & & & & {$[4.4,4.6]$} & {$[3.4,3.6]$} & & \\
\hline
\end{tabular}

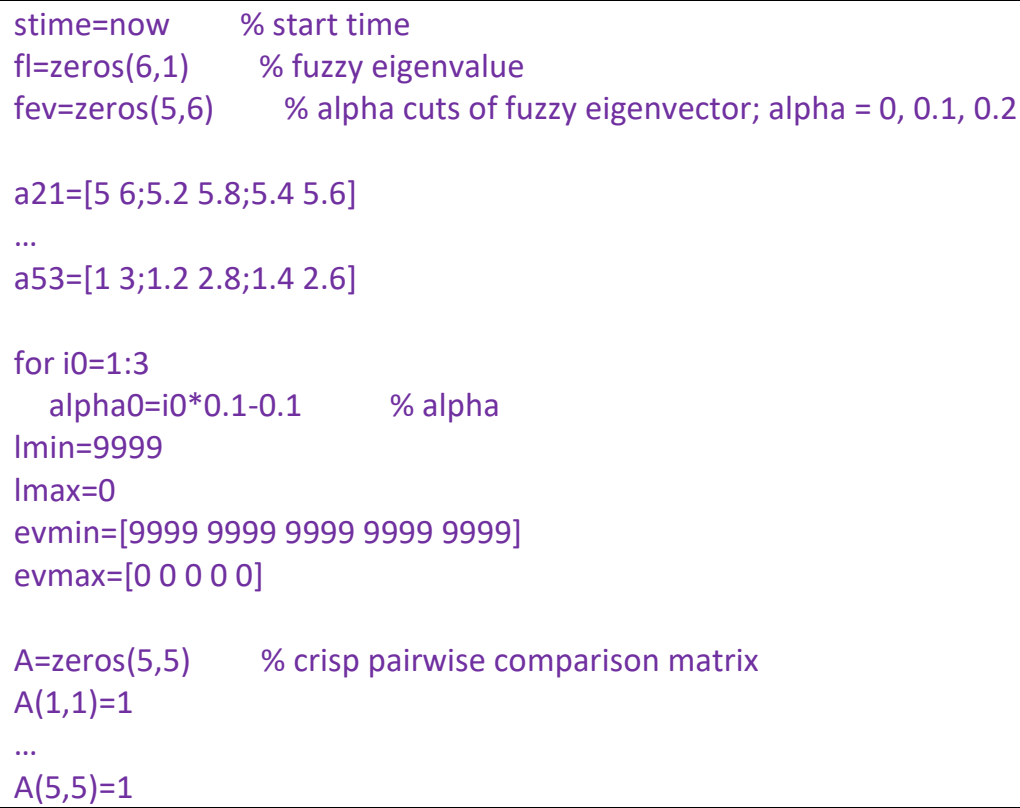




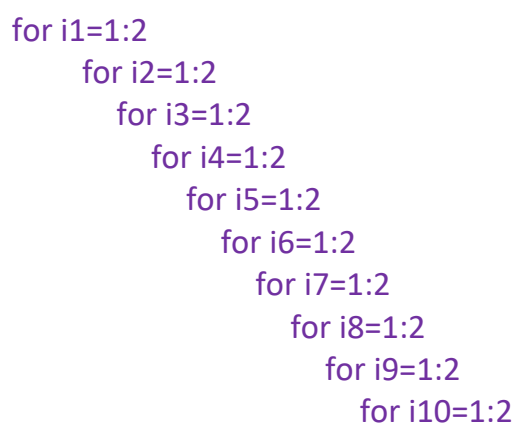




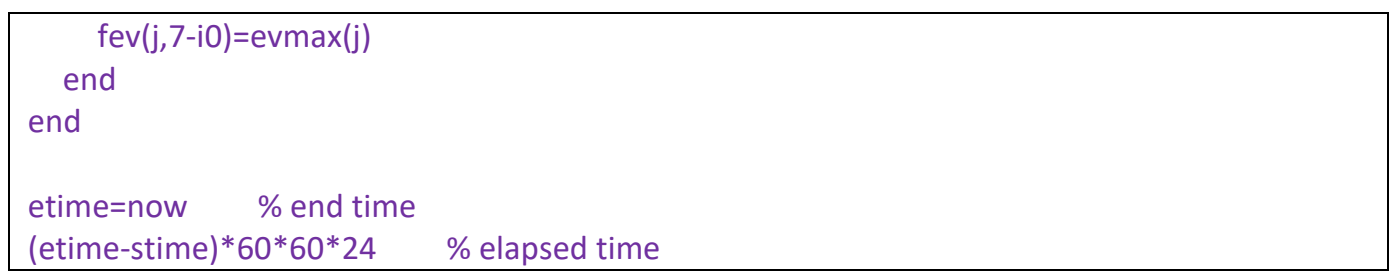

Figure 3. The MATLAB program code.

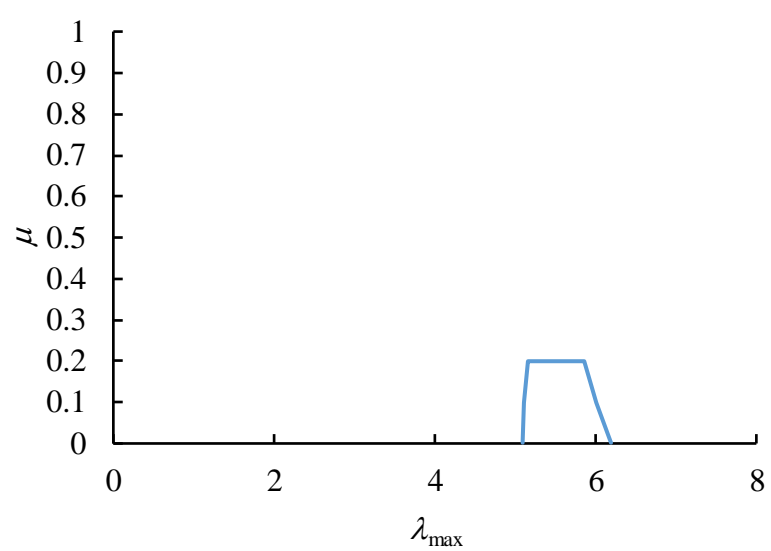

Figure 4. Fuzzy maximal eigenvalue.
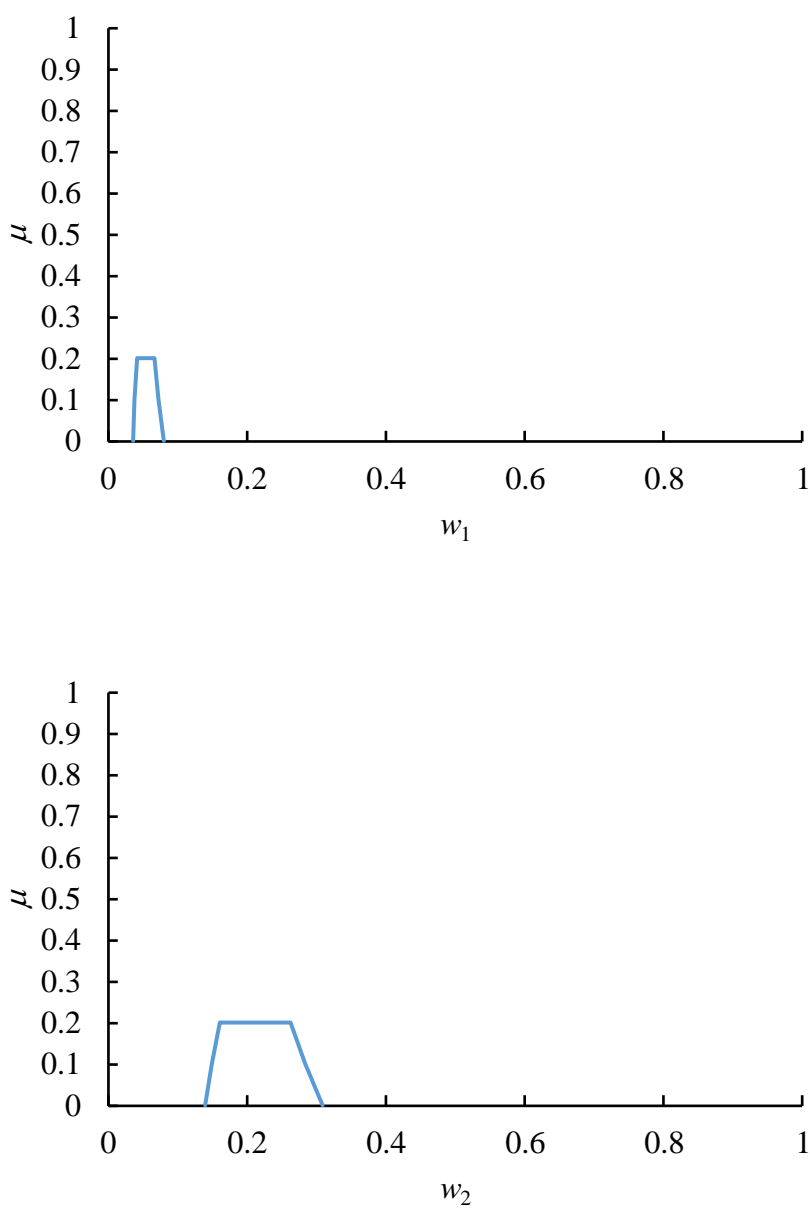

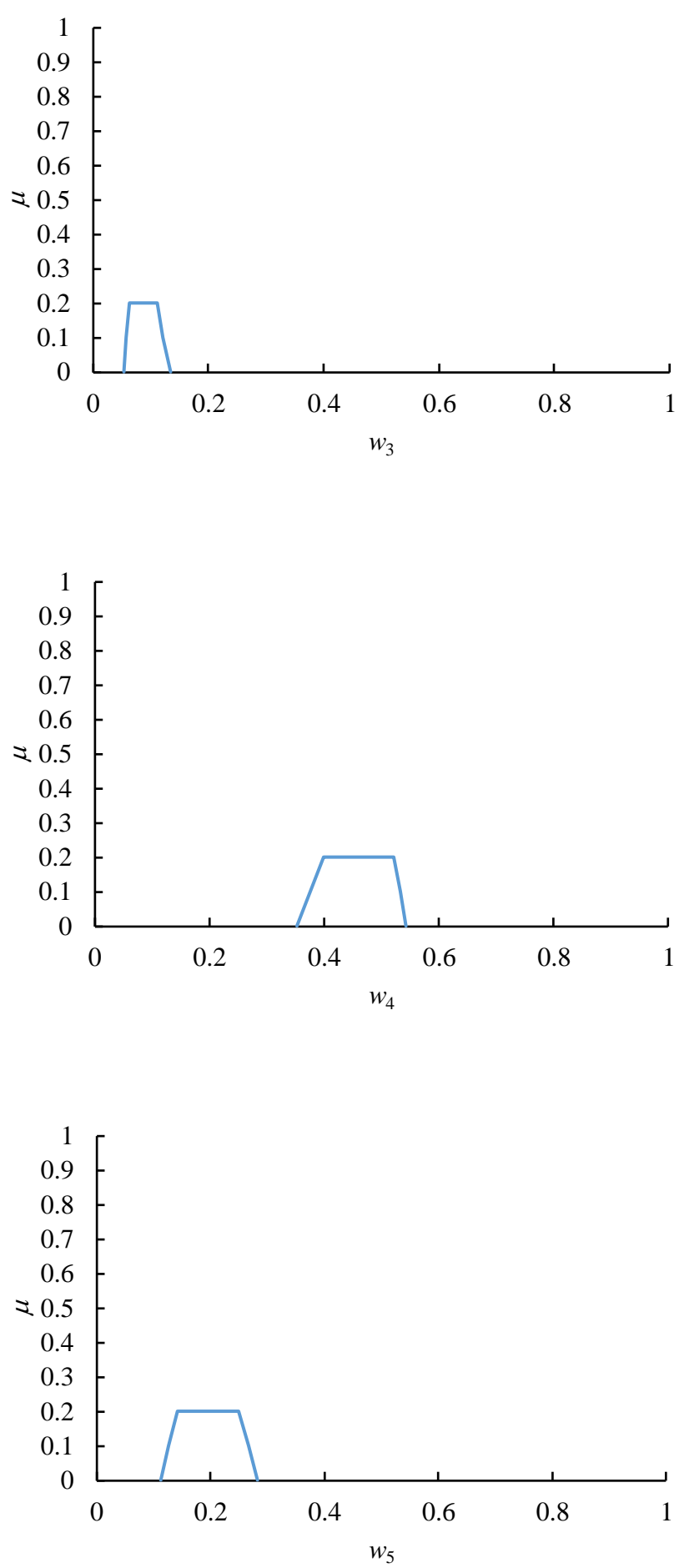

Figure 5. Fuzzy priorities.

\subsection{Application of Existing Methods}

For comparison, three existing methods were also applied to this case. The first existing method was the pre-aggregation FGM-FGM method, in which FGM was applied to aggregate experts' fuzzy judgment matrixes and then derive the fuzzy priorities of criteria from the aggregation result. The results are shown in Figure 6. 


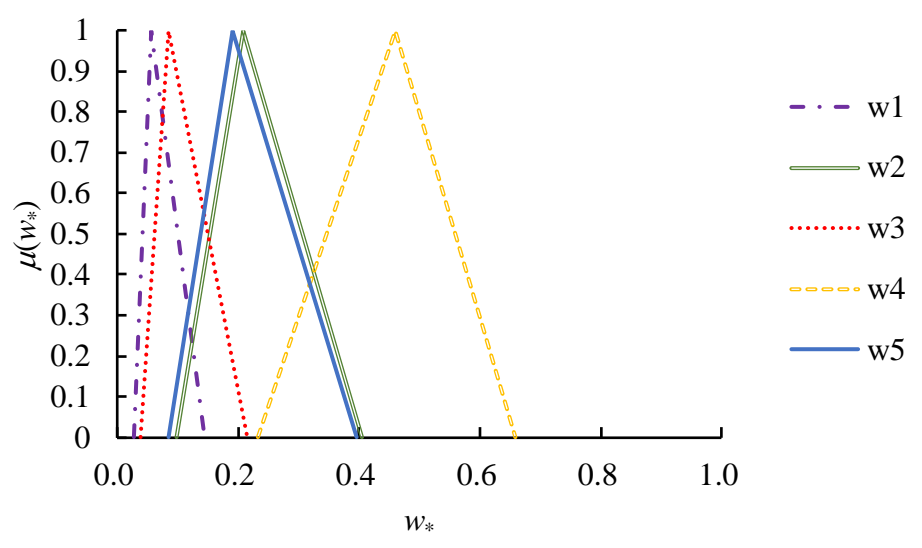

Figure 6. The fuzzy priorities of criteria derived using the FGM-FGM method.

The second existing method was the post-aggregation FGM-fuzzy arithmetic mean (FAM) method, in which each expert derived the fuzzy priorities of criteria using FGM. Then, the fuzzy priorities derived by all experts were aggregated using FAM. The results, shown in Figure 7, were very close to those derived using the FGM-FGM method.

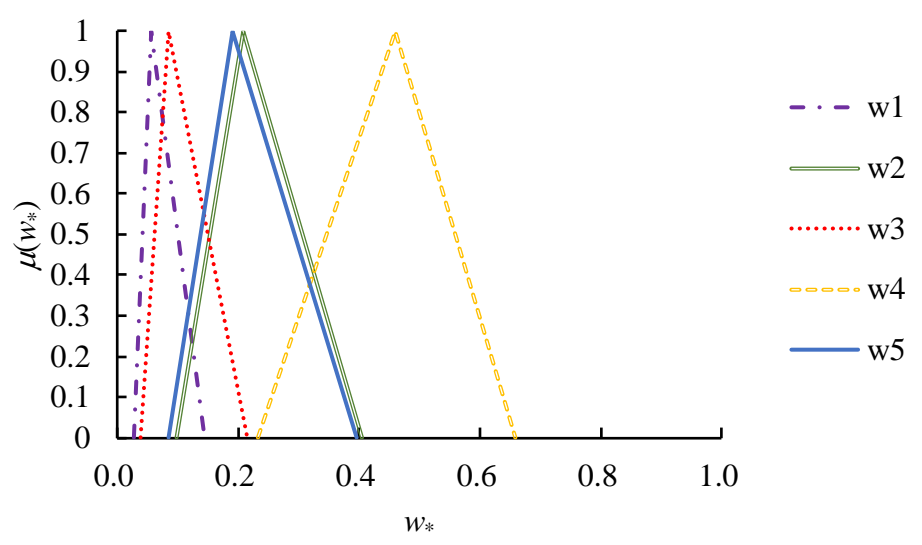

Figure 7. The fuzzy priorities of criteria derived using the FGM-FAM method.

The next compared existing method was the post-aggregation FGM-FI method. First, FGM was applied by each expert to derive the fuzzy priorities of criteria. Then, FI was applied to aggregate the derived fuzzy priorities. The results are shown in Figure 8. The derived fuzzy priorities approximated TFNs. However, the ranges of the fuzzy priorities derived using the FGM-FI method were narrower than those derived by the previous two methods, showing that the precision was improved. In addition, the highest membership of a fuzzy priority derived using the FGM-FI method was usually lower than 1 . 


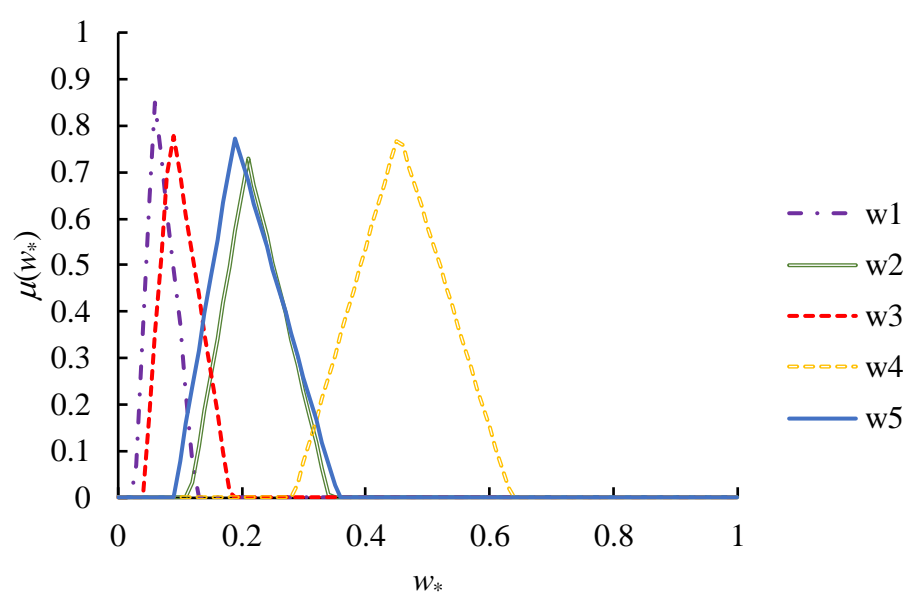

Figure 8. The fuzzy priorities of criteria derived by the post-aggregation FGM-FI method.

\subsection{Discussion}

According to the experimental results, the following points are discussed:

(1) Although $\tilde{a}_{i j}$ 's were given in TFNs, the fuzzy maximal eigenvalue and fuzzy priorities derived using the proposed methodology were polygonal fuzzy numbers with curved edges.

(2) "Pandemic containment performance" was the most critical factor to the performance of an alternative supplier, followed by "delivery speed" and "pandemic severity". In contrast, the least critical factor was "price".

(3) The fuzzy maximal eigenvalue ranged from 5.08 to 6.20, equivalent to a critical ratio of 0.02 to 0.27 . The defuzzified value using the center-of-gravity method [34] was 0.11 , showing sufficient consistency.

(4) By aggregating experts' judgments using the proposed pre-aggregation FCI-based FAHP approach, the uncertainty inherent in the decision-making process has been considerably lessened, which was shown by very narrow fuzzy priorities. For example, the value of $\tilde{w}_{4}$ derived using the proposed methodology is compared with those derived using the existing methods in Figure 9. The proposed methodology shrank the width of $\tilde{w}_{4}$ by up to $55 \%$.

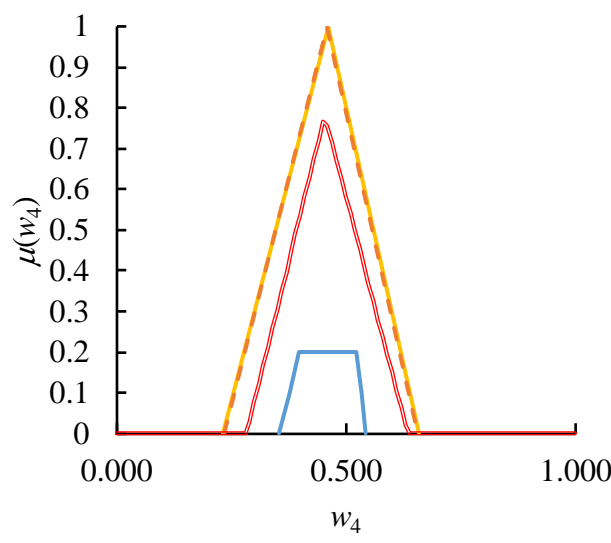

FGM-FGM

--- FGM-FAM

$\longrightarrow$ FGM-FI

- Proposed

Methodology

Figure 9. The values of $\tilde{w}_{4}$ derived using various methods.

\section{Conclusions}


In a fuzzy group decision-making method, experts need to reach a consensus for the decisionmaking result to be acceptable to all of them. However, this requirement has not been fully satisfied by the existing group decision-making FAHP methods. To fill this gap, a pre-aggregation FCI-based FAHP approach is proposed in this study. The pre-aggregation FCI-based FAHP approach satisfies the strictest consensus requirement, i.e., the pairwise comparison results by experts should overlap. In the proposed methodology, first FI is applied to aggregate experts' pairwise comparison results if these pairwise comparison results overlap. The aggregation result is a matrix of polygonal fuzzy numbers. Subsequently, ACO are applied to derive the fuzzy priorities of criteria from the aggregation result.

The pre-aggregation FCI-based FAHP approach has been applied to select suitable alternative suppliers for a wafer foundry in Taiwan amid the COVID-19 pandemic. For elaborating the effectiveness of the pre-aggregation FCI-based FAHP approach, three existing methods were also applied to the same case, so as to make a comparison. Finally, the following conclusions were drawn from the experimental results:

(1) "Pandemic containment performance" and "delivery speed" were considered as the most critical factors to the performance of an alternative supplier, which was obviously due to the COVID-19 pandemic.

(2) Compared to the three existing FAHP methods, the pre-aggregation FCI-based FAHP approach significantly reduced the uncertainty inherent in the decision-making process by deriving fuzzy priorities with very narrow ranges.

The proposed methodology can be extended in future studies to deal with cases in which only a partial consensus has been achieved. In other words, some pairwise comparison results overlap, while others do not. Or, the derived fuzzy priorities overlap, but pairwise comparison results do not.

Author Contributions: Data curation, methodology and writing original draft: H.-C.W., T.-C.T.C.; writingreview and editing: H.-C.W. and T.-C.T.C. Both authors contributed equally to the writing of this paper. Both authors read and approved the final manuscript.

Funding: This study was partly funded by Ministry of Science and Technology, Taiwan.

Conflicts of Interest: The authors declare no conflict of interest.

\section{References}

1. Saaty, T.L. Axiomatic foundation of the analytic hierarchy process. Manag. Sci. 1986, 32, 841-855.

2. Pan, N.F. Fuzzy AHP approach for selecting the suitable bridge construction method. Automation in Construction 2008, 17(8), 958-965.

3. Chang, D.Y. Applications of the extent analysis method on fuzzy AHP. European Journal of Operational Research 1996, 95(3), 649-655.

4. Csutora, R.; Buckley, J.J. Fuzzy hierarchical analysis: the Lambda-Max method. Fuzzy Sets and Systems 2001, 120(2), 181-195.

5. Zheng, G.; Zhu, N.; Tian, Z.; Chen, Y.; Sun, B. Application of a trapezoidal fuzzy AHP method for work safety evaluation and early warning rating of hot and humid environments. Saf. Sci. 2012, 50, 228-239.

6. Tavana, M.; Zareinejad, M.; Di Caprio, D.; Kaviani, M. A. An integrated intuitionistic fuzzy AHP and SWOT method for outsourcing reverse logistics. Applied Soft Computing 2016, 40, 544-557.

7. Cevik Onar, S.; Oztaysi, B.; Kahraman, C. Strategic decision selection using hesitant fuzzy TOPSIS and interval type-2 fuzzy AHP: a case study. International Journal of Computational Intelligence Systems 2014, 7(5), 1002-1021.

8. Acar, C.; Beskese, A.; Temur, G.T. Sustainability analysis of different hydrogen production options using hesitant fuzzy AHP. International Journal of Hydrogen Energy 2018, 43(39), 18059-18076.

9. Chen, Z:; Yang, W. An MAGDM based on constrained FAHP and FTOPSIS and its application to supplier selection. Mathematical and Computer Modelling 2011, 54(11-12), 2802-2815.

10. Gao, H.; Ju, Y.; Gonzalez, E.D.S.; Zhang, W. Green supplier selection in electronics manufacturing: An approach based on consensus decision making. Journal of Cleaner Production 2019, 118781.

11. Wang, Y.C.; Chen, T.; Yeh, Y.L. Advanced 3D printing technologies for the aircraft industry: A fuzzy systematic approach for assessing the critical factors. Int. J. Adv. Manuf. Technol. 2019, 105, 4059-4069. 
12. Chen, T. Assessing factors critical to smart technology applications in mobile health care-The FGM-FAHP approach. Health Policy Technol. 2020, 9, 194-203.

13. Yuen, K.K.F.; Lau, H.C. A fuzzy group analytical hierarchy process approach for software quality assurance management: Fuzzy logarithmic least squares method. Expert Systems with Applications 2011, 38(8), 1029210302.

14. Büyüközkan, G.; Güleryüz, S. A new integrated intuitionistic fuzzy group decision making approach for product development partner selection. Computers \& Industrial Engineering 2016, 102, 383-395.

15. Wang, Y.C.; Chen, T.C.T. A partial-consensus posterior-aggregation FAHP method-Supplier selection problem as an example. Mathematics 2019, 7, 179.

16. Wu, H.C.; Wang, Y.C.; Chen, T.C.T. Assessing and comparing COVID-19 intervention strategies using a varying partial consensus fuzzy collaborative intelligence approach. Mathematics 2020, 8(10), 1725.

17. Chen, T.C.T.; Wang, Y.C.; Lin, C.W. A fuzzy collaborative forecasting approach considering experts' unequal levels of authority. Appl. Soft Comput. 2020, 106455.

18. Pedrycz, W. Collaborative architectures of fuzzy modeling. Lect. Notes Comput. Sci. 2008, 5050, 117-139.

19. Chen, T.C.T.; Honda, K. Fuzzy Collaborative Forecasting and Clustering: Methodology, System Architecture, and Applications; Springer Nature Switzerland AG: Cham, Switzerland, 2019.

20. Chen, T.; Lin, Y.C. A fuzzy-neural system incorporating unequally important expert opinions for semiconductor yield forecasting. Int. J. Uncertain. Fuzziness Knowl.-Based Syst. 2008, 16, 35-58.

21. Wu, H.C.; Chen, T.; Huang, C.H. A piecewise linear FGM approach for efficient and accurate FAHP analysis: Smart backpack design as an example. Mathematics 2020, 8, 1319.

22. Chen, T.C.T. Guaranteed-consensus posterior-aggregation fuzzy analytic hierarchy process method. Neural. Comput. Appl. 2020, 32, 7057-7068.

23. Chen, T.C.T.; Wang, Y.C.; Lin, Y.C.; Wu, H.C.; Lin, H.F. A fuzzy collaborative approach for evaluating the suitability of a smart health practice. Mathematics 2019, 7(12), 1180.

24. Lin, Y.C.; Wang, Y.C.; Chen, T.C.T.; Lin, H.F. Evaluating the suitability of a smart technology application for fall detection using a fuzzy collaborative intelligence approach. Mathematics 2019, 7, 1097.

25. Yazdani, M.; Chatterjee, P.; Zavadskas, E.K.; Zolfani, S.H. Integrated QFD-MCDM framework for green supplier selection. Journal of Cleaner Production 2017, 142, 3728-3740.

26. Alikhani, R.; Torabi, S. A.; Altay, N. Strategic supplier selection under sustainability and risk criteria. International Journal of Production Economics 2019, 208, 69-82.

27. Hosseini, S.; Morshedlou, N.; Ivanov, D.; Sarder, M.D.; Barker, K.; Al Khaled, A. Resilient supplier selection and optimal order allocation under disruption risks. International Journal of Production Economics 2019, 213, 124-137.

28. Lima Junior, F.R.; Osiro, L.; Carpinetti L.C.R. A comparison between Fuzzy AHP and Fuzzy TOPSIS methods to supplier selection. Applied Soft Computing 2014, 21, 194-209.

29. Niu, B.; Li, J.; Zhang, J.; Cheng, H.K.; Tan, Y. Strategic analysis of dual sourcing and dual channel with an unreliable alternative supplier. Production and Operations Management 2019, 28(3), 570-587.

30. Chen, T.; Lin C.-W. Smart and automation technologies for ensuring the long-term operation of a factory amid the COVID-19 pandemic: An evolving fuzzy assessment approach. International Journal of Advanced Manufacturing Technology 2020, in press.

31. Kilpatrick, J. COVID-19: Managing Supply Chain Risk and Disruption. Available online: https://www2.deloitte.com/global/en/pages/risk/articles/covid-19-managing-supply-chain-risk-anddisruption.html (accessed on 7 October 2020).

32. Keegan, K. COVID-19: Operations and Supply Chain Disruption. Available online: https://www.pwc.com/us/en/library/covid-19/supply-chain.html (accessed on 7 October 2020).

33. Virusncov.com. COVID-19 Coronavirus-Update. Available online: https://virusncov.com/ (accessed on 7 October 2020).

34. van Broekhoven, E.; De Baets, B. Fast and accurate center of gravity defuzzification of fuzzy system outputs defined on trapezoidal fuzzy partitions. Fuzzy Sets Syst. 2006, 157, 904-918. 